\title{
MKL1-184C $>$ T gene polymorphism is associated with coronary artery disease in the Chinese Han population
}

Y. Xu' ${ }^{1}$, B. Li ${ }^{2}$, Y.-Z. Bao ${ }^{3}$, X.-Y. Zhang ${ }^{2}$, Z.-Y. Zhu' ${ }^{1}$, X.-F. Zhao' Y.-N. Shi ${ }^{2}$, D. Li $^{2}$, S.-H. Chen ${ }^{4}$, Y. Guo ${ }^{4}$ and C.-H. Cui ${ }^{4}$

${ }^{1}$ Department of Cardiology, People's Hospital of Henan Province, Zhengzhou, China

${ }^{2}$ Xinxiang Medical College, Xinxiang, China

${ }^{3}$ Department of Eye Institute, People's Hospital of Henan Province, Zhengzhou, China

${ }^{4}$ Zhengzhou University, Zhengzhou, China

Corresponding author: Y. Xu

E-mail: yuxucn@126.com

Genet. Mol. Res. 13 (1): 590-597 (2014)

Received December 5, 2012

Accepted September 19, 2013

Published January 28, 2014

DOI http://dx.doi.org/10.4238/2014.January.28.4

\begin{abstract}
We investigated genetic susceptibility to coronary artery disease (CAD) by studying the association of MKL1 gene polymorphisms with $\mathrm{CAD}$ in the Chinese Han population. We performed a case-control study with 476 unrelated CAD patients and 325 non-CAD controls. All SNPs were genotyped with a TaqMan SNP genotyping assay. The distribution of MKL1-184C $>\mathrm{T}$ gene polymorphism in each group was in Hardy-Weinberg equilibrium. The frequency of the MKL1 T allele in the CAD group was significantly higher than in the control group (38.6vs $30.8 \%$ ). After logistic regression models adjusted for CAD risk factors, the risk of CAD among CT genotypes was 1.765 times higher than among the $\mathrm{CC}$ genotypes [odds ration $(\mathrm{OR})=1.765,95 \%$ confidence interval $(\mathrm{CI})=1.246-2.5]$, and for TT genotypes it was 1.806 times higher than for the $\mathrm{CC}$ genotypes $(\mathrm{OR}=1.806,95 \% \mathrm{CI}=1.203-2.71)$.
\end{abstract}


In summary, genotypes with at least one $\mathrm{T}$ allele (CT or TT genotypes) had a significantly increased CAD risk than the $\mathrm{CC}$ genotypes, with a ratio of 1.78 to $1(\mathrm{OR}=1.780,95 \% \mathrm{CI}=1.311-2.418)$. There was a close association between $-184 \mathrm{~T}$ allele and $3 \mathrm{VD}(\mathrm{OR}=1.614,95 \% \mathrm{CI}$ $=1.259-2.07, \mathrm{P}<0.05)$. We conclude that the $-184 \mathrm{C}>\mathrm{T}$ of MKL1 is an important susceptibility factor for CAD in the Han Chinese in Henan Province. Homozygosity for the T allele is not only associated with an increased risk for CAD, it is also correlated with severity of stenosis in the Chinese Han population.

Key words: MKL1; Gene polymorphism; Coronary artery disease

\section{INTRODUCTION}

At present, the medical profession has reached a consensus that many human diseases are the result of the interaction between the environment and the body. Under the same conditions, the differences between the individual risks are not exactly the same; one of the main reasons is gene polymorphism. Coronary artery disease (CAD) is due to myocardial injury, which is caused by an imbalance between myocardial blood supply and myocardial demand due to coronary functional or organic lesions, and affected by multiple factors. Its incidence mode is a combination of genetic predisposition and environmental factors. Along with the continuous development of molecular biology, screening for CAD-associated susceptibility genes has become one of the hotspots of coronary heart disease (CHD) research.

Megakaryoblastic leukemia factor-1 (MKL1) is also called myocardin-related transcription factor-A (MRTF-A), a member of the MRTF family. Human MKL1 gene is located on chromosome 22q13 (Ma et al., 2001). According to reports, MKL1 may have effects on the formation of the cardiovascular system and maintenance processes (Cen et al.., 2004) through the regulation of abnormal proliferation of smooth muscle cells (Sata et al., 2002; Selvaraj and Prywes, 2003; Du et al., 2004; Parmacek, 2007; Kihara et al., 2008), as well as through RhoA and TGF-beta-dependent channels (Elberg et al., 2008) participating in coronary heart disease and its development. Hinohara et al. (2009) first discovered the $-184 \mathrm{C}>\mathrm{T}$ polymorphism of MKL1 involved in the pathogenesis of coronary heart disease of Japanese and Koreans. Therefore, the relationship between MKL1 gene polymorphism and coronary heart disease cannot be ignored. But till now, there are no reports related to the MKL1 gene and coronary heart disease in China. Therefore, this study examined the potential correlation between MKL1 gene polymorphism and genetic predisposition to coronary heart disease in the Han population of Henan, China.

\section{MATERIAL AND METHODS}

\section{Study population}

We selected hospitalized patients in the Henan Provincial People's Hospital, Department of Cardiology from October 2009 to November 2010. All patients with cardiomyopathy, bleeding disorders, renal failure, and cancer and other malignant diseases were excluded. All 
participants underwent coronary angiography and were divided into the case group and control group, according to angiographic results. 1) The case group consisted of 476 cases, 299 males and 177 females, aged 29-83 years old with an average age \pm SD of $61.19 \pm 10.84$. Coronary angiography confirmed that at least one, including the left anterior descending, left circumflex or right coronary artery, showed coronary stenosis $\geq 50 \%$. 2) The control group consisted of 325 cases, 176 males and 149 females, aged 20-89 years old with an average age of $61.10 \pm 12.94$, and coronary angiography ruled out CHD. All participants were genetically unrelated Chinese Han subjects and provided informed consent.

\section{Data collection}

A unified design questionnaire was used to collect detailed data, including age, gender, ethnicity, personal history, family history, smoking history, history of hypertension, diabetes mellitus, history of impaired glucose tolerance, and hypertension and diabetes treatment history, as well as admission records of renal function, liver function, blood lipids, fasting glucose and coronary angiographic findings and discharge diagnosis.

CAD is defined as angiographic evidence of at least one segment of a major coronary artery, including the left anterior descending, left circumflex or right coronary artery with $\geq 50 \%$ organic stenosis. Hypertension is defined as systolic blood pressure (SBP) $\geq 140 \mathrm{mmHg}$ and/or diastolic blood pressure (DBP) $\geq 90 \mathrm{mmHg}$ or taking anti-hypertensive medication. Diabetes was defined as ongoing therapy for diabetes or a fasting plasma glucose (FPG) $\geq 7.0$ $\mathrm{mM}$, or glycosylated hemoglobin of $\geq 6.5 \%$.

\section{Main reagents and instruments}

\section{Main reagents}

The following were commercially obtained: Human lymphocyte separation fluid (Tianjin Hao Yang Biological Manufacture Co., Ltd.); Genomic DNA extraction kit, DNA marker, TaqMan Universal PCR Master Mix (Shanghai GeneCore BioTechnologies Co., Ltd.); General TaqMan probe (designed and synthesized by Shanghai GeneCore BioTechnologies Co., Ltd.): probe1: FAM- AGGCCGCCGTTTTCGCCTT; probe2: HEX-AGGCCGCCGTCT TCGCCTT; primers TAKARA BIOTECHNOLOGY (DALIAN) Co., Ltd.): upstream primer: F: 5'-GTGCCGTCAGTCACAGGAAGT-3'; downstream primers: R: 5'-AAGACTGTCCGC TGGAGAAGT-3'; 10X buffer (Tiangen Biochemical Technology (Beijing) Co., Ltd.).

\section{Main instruments} tems $\mathrm{ABI})$.

Step One Plus Fluorescent real-time quantitative PCR instrument (Applied Biosys-

\section{Genomic DNA extraction}

All research subjects after admission provided a fasting venous blood sample of 2 $\mathrm{ml}$ collected in a heparin anticoagulation tube in the morning. Human lymphocyte separa- 
tion liquid was used to isolate the lymphocytes, and a commercial extraction kit was used for genomic DNA extraction. The extracted genomic DNA was submitted to gel electrophoresis to verify the presence of a single band under a UV lamp, indicating that the genomic DNA obtained was good, and the effectively extracted genomic DNA was then placed in a $-80^{\circ} \mathrm{C}$ freezer till use.

\section{Genotyping}

Polymorphism was detected by the TaqMan MGB probe technology. The total PCR reaction system was $25 \mu \mathrm{L}$, containing $12.5 \mu \mathrm{L}$ TaqMan Universal PCR Master Mix, $0.5 \mu \mathrm{L}$ primer, $0.5 \mu \mathrm{L}$ MGB probe, and deionized water added up to a total volume of $25 \mu \mathrm{L}$. Step One Plus was used for genotyping. PCR cycling parameters were as follows: denaturation at $95^{\circ} \mathrm{C}$ for $10 \mathrm{~min}$, and then 40 cycles of denaturation at $95^{\circ} \mathrm{C}$ for $15 \mathrm{~s}$, annealing at $60^{\circ} \mathrm{C}$ for $1 \mathrm{~min}$, and extension at $60^{\circ} \mathrm{C}$ for $30 \mathrm{~s}$. Samples from 28 randomly selected cases were sent to Beijing Luhe Genomics Technology Co., Ltd. for direct sequencing, and the results were found to be consistent with those of Step One Plus.

\section{Statistical analysis}

Statistical analyses were performed with SPSS 17.0. Genotype and allele frequencies were obtained by direct counting. Congruence in the study objects and with Hardy-Weinberg equilibrium and the comparison between a single genotype and the group allele frequency were evaluated by a $\chi^{2}$ test using $\mathrm{R} \times \mathrm{C}$ contingency tables. Besides, relative risk was shown with an odds ratio (OR) and $95 \%$ confidence interval $(\mathrm{CI})$. The quantitative data are reported as mean \pm standard deviation (means $\pm \mathrm{SD}$ ) and the main testing indices were determined by a normality test. For the distribution of the material by skewness of logarithmic conversation to approximate the normal distribution, we used the Student $t$-test. Besides, we used unconditional logistic regression analysis to evaluate the association between CHD and gene polymorphism. When $\mathrm{P}$ was less than 0.05 , the association was considered to be significant.

\section{RESULTS}

\section{Baseline characteristics of the subjects}

The baseline characteristics of the subjects are summarized in Table 1. As expected, there were no significant differences in age distribution between the case and control groups $(\mathrm{P}>0.05)$. Percentages for sex, diabetes, hypertension and smoking were higher in cases than controls $(\mathrm{P}<0.05$, respectively), and the CAD patients in cases had higher serum cholesterol (TC), triglycerides (TG), low-density lipoprotein cholesterol (LDL-C) and lower serum highdensity lipoprotein cholesterol (HDL-C) $(\mathrm{P}<0.05$, respectively) compared to control subjects.

\section{Distribution of the genotype and allele frequencies in patients and controls}

The distributions of the genotype and allele frequencies in patients and controls are as follows. After checking the frequency distribution of the MKL1 genotype in the two groups 
of people with Hardy-Weinberg equilibrium, we can see that they were in genetic balance (case group, $\chi^{2}=19.36, \mathrm{P}>0.05$; control group, $\chi^{2}=43.16, \mathrm{P}>0.05$ ), thus showing that this group crowd had the group representative and there was no choice bias in selecting the case group and control group. The case group and control group showed a significant difference in MKL1-184 C/T genotype distribution $(\mathrm{P}=0.007)$, and $\mathrm{C}$, $\mathrm{T}$ allele frequency was also significantly different in the two groups $(\mathrm{P}=0.001)$ : $\mathrm{T}$ allele frequency in the case group (38.6 vs $30.8 \%$ ) was significantly higher than in the control group (Table 2).

Table 1. Baseline characteristics of the study population.

\begin{tabular}{lccc}
\hline Characteristics & Cases & Controls & P value \\
\hline Age (years) & $61.19 \pm 10.84$ & $61.10 \pm 12.94$ & 0.919 \\
Male gender [N (\%)] & $299(62.81)$ & $176(54.15)$ & 0.014 \\
Hypertension [N (\%)] & $293(61.55)$ & $160(49.23)$ & 0.001 \\
Diabetes [N (\%)] & $177(37.18)$ & $68(20.92)$ & 0.000 \\
Smoking [N (\%)] & $214(44.96)$ & $4.28 \pm 1.00$ & 0.000 \\
CHOL (mM) & $4.45 \pm 1.04$ & $1.54 \pm 1.11$ & 0.024 \\
TG (mM) & $1.74 \pm 1.18$ & $1.25 \pm 0.39$ & 0.018 \\
HDL-C (mM) & $1.18 \pm 0.44$ & $2.54 \pm 0.73$ & 0.020 \\
LDL-C (mM) & $2.73 \pm 0.80$ & 0.001 \\
\hline
\end{tabular}

$\mathrm{N}=$ number of cases. $\mathrm{CHOL}=$ total cholesterol; $\mathrm{TG}=$ triglycerides; HDL-C $=$ high-density lipoprotein cholesterol; LDL-C $=$ low-density lipoprotein cholesterol. Age, CHOL, TG, HDL-C, and LDL-C are reported as means \pm SD..

\section{Analysis of relevance between MKL-184C $>$ T and CHD}

The analysis of relevance between MKL-184C $>\mathrm{T}$ and CHD was as follows. Relative risk analysis of $\mathrm{T}$ alleles showed $\mathrm{OR}=1.412(95 \% \mathrm{CI}=1.142-1.744, \mathrm{P}=0.001)$ and that there was a signification correlation with susceptibility to CHD. A regression model was established with whether suffering from coronary heart disease as the dependent variable and sex, history of smoking, coronary heart disease and diabetes, serum TC, TG and LDL-C and HDL-C, and MKL1 polymorphic genotypes as the independent variable to carry out logistic regression analysis. The results showed that after evaluating other risk factors for CHD effects, these differences still showed remarkable significance and MKL1 polymorphic genotypes were closely related to CHD. The risk of CAD among CT genotypes was 1.765 times higher than that of CC genotypes $(\mathrm{OR}=1.765,95 \% \mathrm{CI}=1.246-2.5)$, and TT genotypes showed 1.806 times higher risk than $\mathrm{CC}$ genotypes $(\mathrm{OR}=1.806,95 \% \mathrm{CI}=1.203-2.71)$. $\mathrm{CT}+\mathrm{TT}$ genotypes had a significantly increased CAD risk compared to CC genotypes with a ratio of $1.78(\mathrm{OR}=1.78,95 \% \mathrm{CI}$ $=1.311-2.418)$ (Table 3).

Table 2. CHD group and control group of MKL1 genotype and allele frequency distribution.

\begin{tabular}{lccccc}
\hline Groups & \multicolumn{3}{c}{ Genotype } & & Allele \\
\cline { 2 - 4 } & $\mathrm{CC}$ & $\mathrm{CT}$ & $\mathrm{TT}$ & $\mathrm{C}$ & $\mathrm{T}$ \\
\hline Cases $(\mathrm{N}=476)$ & $211(44.3)$ & $16(34.2)$ & $102(21.4)$ & $585(61.4)$ & $367(38.6)$ \\
Controls $(\mathrm{N}=325)$ & $181(55.7)$ & $88(27.1)$ & $56(17.2)$ & & $450(69.2)$ \\
$\chi^{2}$ & 9.988 & & & 10.228 & $200(30.8)$ \\
$\mathrm{P}$ & 0.007 & & & 0.001 \\
\hline
\end{tabular}




Table 3. Risk relationship of MKL1 genotype and coronary heart disease.
\begin{tabular}{lrrrrr}
\hline Genetype & \multicolumn{2}{c}{ No. (frequency) } & OR & $95 \%$ CI & P \\
\cline { 2 - 4 } & Cases & Controls & & & \\
\hline CC & $211(0.443)$ & $181(0.557)$ & 1 & & \\
CT & $163(0.342)$ & $88(0.271)$ & 1.765 & $1.246-2.500$ & 0.001 \\
TT & $102(0.214)$ & $56(0.172)$ & 1.806 & $1.203-2.710$ & 0.004 \\
CT+TT & $265(0.557)$ & $144(0.443)$ & 1.78 & $1.311-2.418$ & 0.000 \\
\hline
\end{tabular}

\section{Correlation between the genotype and severity of coronary atherosclerosis}

The correlation between the genotype and severity of coronary atherosclerosis is shown in Table 4. According to the number of significantly involved vessels, the patients were classified into four groups, $0 \mathrm{VD}, 1 \mathrm{VD}, 2 \mathrm{VD}$ and $3 \mathrm{VD}$. There was a strong association between $-184 \mathrm{~T}$ allele and $3 \mathrm{VD}(\mathrm{OR}=2.877,95 \% \mathrm{CI}=1.831-4.523, \mathrm{P}<0.05)($ Table 4$)$.

Table 4. Association of -184 T allele of MKL1 with the severity of CAD.
\begin{tabular}{lccrr}
\hline Groups & $\mathrm{C}$ & $\mathrm{T}$ & $\mathrm{OR}(95 \% \mathrm{CI})$ & $\mathrm{P}$ \\
\hline Zero-VD [N (\%)] & $450(69.2)$ & $200(30.8)$ & 1 & \\
One-VD [N (\%)] & $193(65.2)$ & $103(34.8)$ & $1.201(0.897-1.607)$ & 0.219 \\
Two-VD [N (\%)] & $123(63.4)$ & $71(36.6)$ & $1.299(0.928-1.818)$ & 0.128 \\
Three-VD [N (\%)] & $269(58.2)$ & $193(41.8)$ & $1.614(1.259-2.070)$ & 0.000 \\
\hline
\end{tabular}

\section{DISCUSSION}

With the rapid development of molecular biology technology, scientists have found many gene polymorphisms closely related to coronary heart disease susceptibility, such as those of angiotensin converting enzyme (Cambien et al., 1992; Narne et al., 2012), apolipoprotein (Hsu et al., 2006; Jang et al., 2009; Huang et al., 2012), cell factor (Zhong et al., 2009; Fragoso et al., 2010; Jin et al., 2012; Srikanth Babu et al., 2012), coagulation system (Pegoraro, 2005; Friso et al., 2012), and endothelin (Popov et al., 2008).

MKL1, also known as MRTF-A, is a member of MRTF family. Human MKL1 gene is located on chromosome 22q13 (Ma et al., 2001) and encodes a 931-amino acid protein. Hinohara et al. (2009) found that $-184 \mathrm{C}>\mathrm{T}$ of MKL1 is involved in the pathogenesis of CAD in the Japanese and Korean populations: the risk was 1.25 times higher for people who carried the $\mathrm{T}$ allele versus the $\mathrm{C}$ allele in the Japanese population $(\mathrm{OR}=1.25,95 \% \mathrm{CI}=1.04-1.49, \mathrm{P}=$ $0.015)$, while the value was 1.26 in South Korea $(\mathrm{OR}=1.26,95 \% \mathrm{CI}=1.01-1.58, \mathrm{P}=0.042)$. The study also revealed that the SNP conferred high expression of MKL1, and the T allele homozygotes showed about 1.8-fold higher expression than the $\mathrm{C}$ allele homozygotes. The gene polymorphism of the MKL1-184C $>\mathrm{T}$ has racial and geographic differences. The association between MKL1 gene polymorphisms and CAD in the Chinese Han population has not been previously reported. In our study, we chose the Chinese Han population as research subjects to identify the potential association between MKL1 gene polymorphisms and CAD.

In this study, we found a significant difference in MKL1-184C $>\mathrm{T}$ genotype frequency distribution between the case group and control group. $\mathrm{T}$ allele frequency in the case group 
was significantly higher than in the control group, and it had a significant correlation with CHD. After adjusting the effects from Traditional risk factors to the CHD with logistic regression analysis, we obtained the following results. The risk of CAD among CT genotypes was 1.765 times higher than that of CC genotypes, and TT genotypes showed 1.806 times higher risk than $\mathrm{CC}$ genotypes. In summary, genotypes with at least one $\mathrm{T}$ allele (CT or TT genotypes) had a significantly increased CHD risk compared to the CC genotypes with a ratio of 1.78. Because of the sample size effect or difference in region, race and standards in choosing the cases, the differences between the case groups and the control groups were higher compared to the results reported by Hinoharak et al. (2009), but the finding that the T allele may be an independent risk factor for CHD was in accordance with their report. In addition, we also found that there was a close correlation between -184 T allele and 3VD.

From the above results, we can clearly see that $-184 \mathrm{C}>\mathrm{T}$ of MKL1 was an important susceptibility locus of CAD in the Chinese Han population. Homozygosity was associated with an increased risk for $\mathrm{CAD}$ in the Chinese Han population, which suggests a potential role of MKL1 in the pathogenesis of CAD. According to these findings, MKL1 may affect the developmental process of CHD through the following aspects. First, MKL1 can convert the signals from the cytoskeleton of smooth muscle cells to nucleic acid, alone or together with serum response factor to regulate the differentiation of myocardial protein-dependent smooth muscle cells to affect smooth muscle cell proliferation (Du et al., 2004). Also, the excessive accumulation of SMCs plays an important role in the pathogenesis of atherosclerosis (Sata et al., 2002). Second, myocardial protein plays an important role during the development of the heart, and as one of the myocardin-related transcription factors. Parmacek found that MKL1 null mice were born at a rate lower than that predicted by Mendelian frequency, and $35 \%$ death of MKL1 null embryos was due to dilated cardiac chambers, aorta, and pulmonary arteries accompanied by heart failure. Also, approximately $40 \%$ of MKL1 null mice embryos suffered from lethal cardiac cell necrosis with mitochondrial dysfunction (Parmacek, 2007). In addition, it has been reported that the expression of MKL1 in the smooth muscle cells may be associated with RhoA and TGF-beta-dependent channels, and that these two channels have an important role in the process of atherosclerosis (Elberg et al., 2008). Hence, we hypothesized that MKL1 may also play an important role in coronary atherosclerosis.

In conclusion, we found that $-184 \mathrm{C}>\mathrm{T}$ of MKL1 is an important susceptibility locus of CAD in the Chinese Han population. The T allele is not only a genetic risk factor for CAD but is also correlated with severity of stenosis in the Chinese Han population. These findings may permit targeting prevention and early intervention in high-risk patients to reduce their cardiovascular risk.

\section{REFERENCES}

Cambien F, Poirier O, Lecerf L, Evans A, et al. (1992). Deletion polymorphism in the gene for angiotensin-converting enzyme is a potent risk factor for myocardial infarction. Nature 359: 641-644.

Cen B, Selvaraj A and Prywes R (2004). Myocardin/MKL family of SRF coactivators: key regulators of immediate early and muscle specific gene expression. J. Cell Biochem. 93: 74-82.

Du KL, Chen M, Li J, Lepore JJ, et al. (2004). Megakaryoblastic leukemia factor-1 transduces cytoskeletal signals and induces smooth muscle cell differentiation from undifferentiated embryonic stem cells. J. Biol. Chem. 279: 1757817586 .

Elberg G, Chen L, Elberg D, Chan MD, et al. (2008). MKL1 mediates TGF-beta1-induced alpha-smooth muscle actin expression in human renal epithelial cells. Am. J. Physiol. Renal Physiol. 294: F1116-F1128. 
Fragoso JM, Delgadillo H, Llorente L, Chuquiure E, et al. (2010). Interleukin 1 receptor antagonist polymorphisms are associated with the risk of developing acute coronary syndrome in Mexicans. Immunol. Lett. 133: 106-111.

Friso S, Lotto V, Choi SW, Girelli D, et al. (2012). Promoter methylation in coagulation F7 gene influences plasma FVII concentrations and relates to coronary artery disease. J. Med. Genet. 49: 192-199.

Hinohara K, Nakajima T, Yasunami M, Houda S, et al. (2009). Megakaryoblastic leukemia factor-1 gene in the susceptibility to coronary artery disease. Hum. Genet. 126: 539-547.

Hsu LA, Ko YL, Chang CJ, Hu CF, et al. (2006). Genetic variations of apolipoprotein A5 gene is associated with the risk of coronary artery disease among Chinese in Taiwan. Atherosclerosis 185: 143-149.

Huang G, Zhong H, Re HM, Mao HW, et al. (2012). Coalition of DNA polymorphisms of ApoB and ApoAI genes is related with coronary artery disease in Kazaks. J. Geriatr. Cardiol. 9: 33-37.

Jang Y, Paik JK, Hyun YJ, Chae JS, et al. (2009). The apolipoprotein A5 -1131T >C promoter polymorphism in Koreans: association with plasma APOA5 and serum triglyceride concentrations, LDL particle size and coronary artery disease. Clin. Chim. Acta 402: 83-87.

Jin KK, Xu F, Xu LX, Zhang HQ, et al. (2012). Association between polymorphisms of inflammatory factor genes and coronary heart disease. Zhonghua Yi Xue Yi Chuan Xue Za Zhi. 29: 200-205.

Kihara T, Kano F and Murata M (2008). Modulation of SRF-dependent gene expression by association of SPT16 with MKL1. Exp. Cell Res. 314: 629-637.

Ma Z, Morris SW, Valentine V, Li M, et al. (2001). Fusion of two novel genes, RBM15 and MKL1, in the t(1;22)(p13;q13) of acute megakaryoblastic leukemia. Nat. Genet. 28: 220-221.

Narne P, Ponnaluri KC, Singh S, Siraj M, et al. (2012). Relationship between angiotensin-converting enzyme gene insertion/deletion polymorphism, angiographically defined coronary artery disease and myocardial infarction in patients with type 2 diabetes mellitus. J. Renin Angiotensin Aldosterone Syst. 13: 478-486.

Parmacek MS (2007). Myocardin-related transcription factors: critical coactivators regulating cardiovascular development and adaptation. Circ. Res. 100: 633-644.

Pegoraro RJ, Ranjith N and Rom L (2005). Coagulation gene polymorphisms as risk factors for myocardial infarction in young Indian Asians. Cardiovasc. J. S. Afr. 16: 152-157.

Popov AF, Schulz EG, Hinz J, Schmitto JD, et al. (2008). Impact of endothelin-1 Lys198Asn polymorphism on coronary artery disease and endorgan damage in hypertensives. Coron. Artery Dis. 19: 429-434.

Sata M, Saiura A, Kunisato A, Tojo A, et al. (2002). Hematopoietic stem cells differentiate into vascular cells that participate in the pathogenesis of atherosclerosis. Nat. Med. 8: 403-409.

Selvaraj A and Prywes R (2003). Megakaryoblastic leukemia-1/2, a transcriptional co-activator of serum response factor, is required for skeletal myogenic differentiation. J. Biol. Chem. 278: 41977-41987.

Srikanth Babu BMV, Pulla Reddy B, Sai Priya VH, Munshi A, et al. (2012). Cytokine gene polymorphisms in the susceptibility to acute coronary syndrome(J). Genet. Test Mol. Biomarkers 16: 359-365.

Zhong C, Quanzhong Y, Genshan M, Hua Z, et al. (2009). Myeloperoxidase gene-463G > A polymorphism and premature coronary artery disease. Genet. Mol. Biol. 32: 260-263. 